\title{
Schlaganfall
}

\section{Hirnblutungen unter Antikoagulanzien}

Studie zur Akut- und Langzeittherapie von Patienten mit Hirnblutung, die mit Antikoagulanzien therapiert wurden.

Experten aus 19 deutschen Universitätskliniken und Krankenhäusern haben über 5 Jahre hinweg die Daten von über 1000 Schlaganfallpatienten ausgewertet und diejenigen Patienten neurologisch nachbeobachtet, die mit Blutverdünnungsmittel therapiert wurden. Die Ergebnisse dieser bislang größten Studie [1] zum Thema „Blutverdünner und Hirnblutung" wurden kürzlich im „Journal of the American Medical Association" veröffentlicht. Die Analyse untersuchte einerseits verschiedene Akuttherapien zur Normalisierung der gestörten Gerinnung und zum Blutdruckmanagement, andererseits wurde das Auftreten von erneuten Hirnblutungen oder Schlaganfällen während des einjährigen Nachbeobachtungszeitraums in Abhängigkeit von einer erneuten Blutverdünnung verglichen.

Studienleiter Prof. Dr. Hagen B. Huttner und Erstautor Dr. Joji Kuramtasu, von der Neurologischen Klinik des Universitätsklinikums Erlangen, fassen zusammen: „Für die Akutbehandlung konnten wir feststellen, dass eine rigorose Normalisierung der Blutgerinnung durch Aufhebung der medikamentösen Blutverdünnungswirkung am wenigsten zu einer Nachblutung führte." Dieser Effekt konnte zusätzlich gesteigert werden, wenn die systolischen Blutdruckwerte innerhalb der ersten Stunden unter $160 \mathrm{mmHg}$ lagen.

Im zweiten Teil der Studie verglichen die Forscher all jene Patienten, die im Therapieverlauf erneut mit Blutverdünnern behandelt wurden, mit einer sta- tistisch vergleichbaren Kontrollgruppe ohne Blutverdünner. Dabei wurde über ein Jahr hinweg die Häufigkeit von Schlaganfällen erfasst, sowohl solche, die durch Minderdurchblutung entstanden, als auch erneute Hirnblutungen. Die Wiederaufnahme der Blutverdünnung zeigte einen klaren Schutz vor Schlaganfällen, ohne gleichzeitig das erneute Auftreten der gefürchteten Hirnblutung zu begünstigen. Somit ergab sich ein Netto-Nutzen zugunsten der Wiederaufnahme der „Blutverdünnung."

\section{DGN/Universitätskli-}

nikum Erlangen

Literatur:

1. Kuramatsu JB et al (2015) Anticoagulant reversal, blood pressure levels, and anticoagulant resumption in patients with anticoagulation-related intracerebral hemorrhage. JAMA 313(8):824-836. doi:10.1001/ jama.2015.0846 psychopraxis.neuropraxis 2015 · 18:63 DOI 10.1007/s00739-015-0248-z Online publiziert: 24. März 2015 C) Springer-Verlag Wien 2015 